\title{
Magnetopause stand-off distance in dependence on the magnetosheath and solar wind parameters
}

\author{
M. I. Pudovkin ${ }^{1}$, B. P. Besser ${ }^{2}$, S. A. Zaitseva ${ }^{1}$ \\ 1 Institute of Physics, University of St. Petersburg, St. Petersburg 198904, Russia \\ 2 Institut für Weltraumforschung, Österreichische Akademie der Wissenschaften, Inffeldgasse 12, A-8010 Graz, Austria
}

Received: 12 November 1996 / Revised: 25 July 1997 / Accepted: 10 September 1997

\begin{abstract}
A model of the magnetosheath structure proposed in a recent paper from the authors is extended to estimate the magnetopause stand-off distance from solar wind data. For this purpose, the relationship of the magnetopause location to the magnetosheath and solar wind parameters is studied. It is shown that magnetopause erosion may be explained in terms of the magnetosheath magnetic field penetration into the magnetosphere. The coefficient of penetration (the ratio of the magnetospheric magnetic field depression to the intensity of the magnetosheath magnetic field $B_{m \perp z}=-B_{m} \sin ^{2} \Theta / 2$, is estimated and found approximately to equal 1 . It is shown that having combined a magnetosheath model presented in an earlier paper and the magnetosheath field penetration model presented in this paper, it is possible to predict the magnetopause stand-off distance from solar wind parameters.
\end{abstract}

Key words. Magnetospheric physics - Magnetopause · Cusp and boundary layers-Magnetosheath

\section{Introduction}

In a recent paper (Pudovkin et al., 1995) the authors proposed a model of the magnetosheath structure which in particular allows one to estimate the intensity of the magnetic field at the magnetopause.

Knowing the magnetosheath parameters in the vicinity of the magnetopause, one can calculate its stand-off distance, thereby transfer from relative spatial coordinates to absolute ones, and essentially advance the model.
On the other hand, the problem of the location of the magnetopause is interesting in itself and has attracted the attention of geophysicists for many years. First of all, this distance may be considered as a natural scale length determining the structure of the magnetosphere. On the other hand, the magnetopause position depends on peculiarities of solar wind - magnetosphere interaction processes and hence bears information on these processes.

The first estimate of the magnetopause location was obtained by Chapman and Ferraro (1931) from a simple model consideration. More sophisticated models by Spreiter and Briggs (1962) and Mead (1964) based on numerical modelling extended the results by Chapman and Ferraro for a three-dimensional case and permitted the authors to obtain the shape of the dayside magnetopause.

According to the Mead (1964) model, the geocentric distance of the subsolar magnetopause equals

$$
r_{0}=\left(\frac{f^{2} M_{E}^{2}}{8 k \pi n m_{p} v^{2}}\right)^{1 / 6}
$$

where $M_{E}$ is the magnetic momentum of the Earth dipole and $m_{p}$ is the proton mass; $f$ determines the amplification of the terrestrial magnetic dipole field by magnetopause currents (in the flat model by Chapman and Ferraro $f=2$; in the three-dimensional model by Mead $f=2.44)$; the parameter $k$ depends on the character of the interaction of the solar wind particles with the magnetopause, and according to Spreiter et al. (1968), $k=0.88$.

Later, Aubry et al. (1970) showed that for a given solar wind pressure, a southward turning of the interplanetary magnetic field (IMF) causes an Earthward motion of the magnetopause. This result was later confirmed by Fairfield (1971), Maezawa (1974), Petrinec et al. (1991), Sibeck et al. (1991), and Tsyganenko and Sibeck (1994), who showed that during periods of southward IMF, the magnetopause is $0.5-1.5 R_{E}$ closer to the Earth than during periods of northward IMF. A close correlation between the magnetopause stand-off distance and the intensity of the IMF $B_{z}$-component was 
revealed by Sibeck et al. (1991) and Petrinec and Russell (1993).

Thus, the Earthward displacement of the dayside magnetopause during southward IMF periods and its relationship with the $Z$-component of the IMF are surely revealed by experimental data. What concerns the nature of that phenomenon, it is as yet not quite understood.

Kovner and Feldstein (1973) interpreted the Earthward displacement of the magnetopause as a result of the magnetosheath magnetic field penetration into the magnetosphere. Their hypothesis was confirmed by the fact that the magnetospheric magnetic field as measured in the vicinity of the subsolar point differed from the value of the field calculated in the frame of the Mead model by the value of the magnetosheath magnetic field. However, they have analyzed data of three crossings only, so that their result seems to be rather dubious from the statistical viewpoint. Their hypothesis was later developed further by Pudovkin (1982) and Pudovkin et al. (1984). In their model, the sheath magnetic field penetration into the magnetosphere was supposed to be associated with magnetic field reconnection at the magnetopause, so that only the field component perpendicular to the merging line could enter the magnetosphere.

The results obtained agreed with experimental data and seemed to confirm the Kovner and Feldstein (1973) hypothesis. However, the amount of experimental data used in their analysis was rather small. Besides, the mechanism of the sheath magnetic field penetration into the dayside magnetosphere was not specified in those papers. In subsequent papers by Pudovkin et al. $(1986 a, b)$ it was supposed that the magnetosheath magnetic field penetration associated with reconnection is caused by the disruption of the magnetopause electric currents and their diversion into the ionosphere along geomagnetic field lines, resulting in the formation of a Birkeland current loop of the McPherron et al. (1973) type in the dayside cusp region.

The field penetration model was criticized by Sibeck et al. (1991) on the basis that in contrast to the magnetosheath field, the much stronger northward magnetospheric field is prohibited from penetrating outward to produce a northward magnetosheath magnetic field. However, this criticism seems to concern the field penetration model in its primary, 25-year-old form, and does not take into account its real physical meaning.

An apparently different explanation of the dayside magnetopause erosion was proposed by Holzer and Slavin $(1978,1979)$. According to their model, the most likely mechanism of that erosion is the transfer of the magnetic flux from the dayside magnetosphere to the magnetotail through the reconnection process, the contraction or expansion of the magnetopause being determined by the imbalance of the reconnection rate at the dayside magnetopause and in the magnetotail. Experimental data presented by the authors convincingly confirm the model. A similar model was later proposed by Petrinec and Russell (1993).

However, a new equilibrium location of the magnetopause which has to be settled after a southward turn of the IMF at the moment when the reconnection rate at the magnetopause and in the magnetotail balance each other, has not been considered by the authors. This may be explained in part by the fact that Holzer and Slavin $(1978,1979)$ do not specify the magnetospheric current system which is responsible for the magnetic field depression in the dayside magnetosphere in their model, while the current system proposed earlier by Holzer and Reid (1975) and by Reid and Holzer (1975) seems to be rather unrealistic.

Sibeck et al. (1991) proposed a model in which the erosion of the dayside magnetosphere is explained by the effect of a Birkeland current loop in the cusp region. By its physical content, this model seems to coincide with that proposed by Pudovkin et al. (1986a, b). In this connection, a conclusion by Tsyganenko and Sibeck (1994) that "the decrease in the outer magnetosphere magnetic field and pressure should be attributed to variations of magnetospheric currents rather than to a penetrating IMF" seems to be based on a difference in terminology rather than physical ideas. It is obvious that any change of the magnetic field may be caused only by the variation of some current system, and we use the term "penetration" only to prolong a tradition and to emphasize a close quantitative relationship between the intensity of the magnetospheric magnetic field depression and the magnetosheath magnetic field.

Tsyganenko and Sibeck (1994) have studied this problem in detail. In particular, they have accurately calculated the magnetic field produced by Birkeland currents observed in the dayside cusp region and by cross-tail currents. As a result, they have shown that the Earthward displacement of the dayside magnetopause observed during southward IMF periods may really be explained by the depression of the magnetospheric magnetic field produced by those two current systems.

Sibeck et al. (1991) studied the relationship between the cusp Birkeland current loop intensity and the IMF $B_{z}$-component and obtained corresponding regression coefficients. This may be greatly useful for prediction of the magnetopause stand-off distance from solar wind data. At the same time, the magnetic field reconnection process (including the generation of Birkeland currents) is determined by local magnetic field and plasma parameters. In this connection, we shall try in this paper, first of all, to reveal a dependence of the magnetopause stand-off distance on the magnetosheath magnetic field intensity and direction, then to estimate the magnetospheric magnetic field depression intensity and to find a relationship with various components of the magnetosheath magnetic field. Then, using the model by Pudovkin et al. (1995), we shall try to relate the magnetopause stand-off distance to the solar wind parameters in front of the bow shock.

\section{Model}

Following the arguments of the preceding section, we shall suppose that the magnetic field reconnection process developing at the magnetopause during south- 
ward IMF periods causes a diversion of a part of the magnetopause currents from the magnetopause to the ionosphere, producing a huge Birkeland current loop of the McPherron et al. (1973) type.

In the vicinity of the subsolar point, the magnetic field of these currents is directed southward as in the magnetosheath, which allows it to be considered, after Kovner and Feldstein (1973), in terms of a partial penetration of the magnetosheath magnetic field (the component perpendicular to the reconnection line) into the dayside magnetosphere.

The pressure balance at the dayside magnetopause may be written as (Pudovkin et al., 1984):

$P_{0}=\frac{B_{0 i}^{2}}{8 \pi}=\frac{1}{8 \pi}\left\{\left[\frac{2.44 \cdot M_{E}^{\prime}}{r_{0}^{3}}+\delta B_{z}\right]^{2}+\delta B_{y}^{2}\right\}$,

where $P_{0}$ is the sum of the plasma and magnetic field pressure in the magnetosheath, $B_{0 i}$ is the magnetospheric magnetic field in the vicinity of the subsolar point; $\delta \mathbf{B}$ is the intensity of the penetrating magnetic field; $r_{0}$ is the magnetopause stand-off distance, and $M_{E}^{\prime}=M_{E}+M_{D R}$ is the sum of the Earth's dipole and DR-current momenta (Schield, 1969a, b).

Concerning the left-hand side of Eq. (2), it may be shown that $P_{0}=P_{d}+P_{T}+P_{B}=P_{d w}$, where $P_{d w}=$ $n_{w} m_{p} v_{w}^{2}$ is the dynamic pressure of the solar wind (Pudovkin et al., 1982).

Let $\mathbf{B}_{\mathbf{m}}$ be the magnetosheath magnetic field and $\Theta$ the angle between $\mathbf{B}_{\mathbf{m}}$ and the $Z$-axis of the GSM coordinate system (Fig. 1). The angle between the reconnection line and the $Z$-axis equals approximately $\Theta / 2$ (Yeh, 1976). Then the $\mathbf{B}_{\mathbf{m}}$ component perpendicular to the merging line equals $B_{m \perp}=B_{m} \sin \Theta / 2$; the $Y$ - and $Z$-components of the magnetic field supposed to penetrate into the magnetosphere equal, correspondingly:

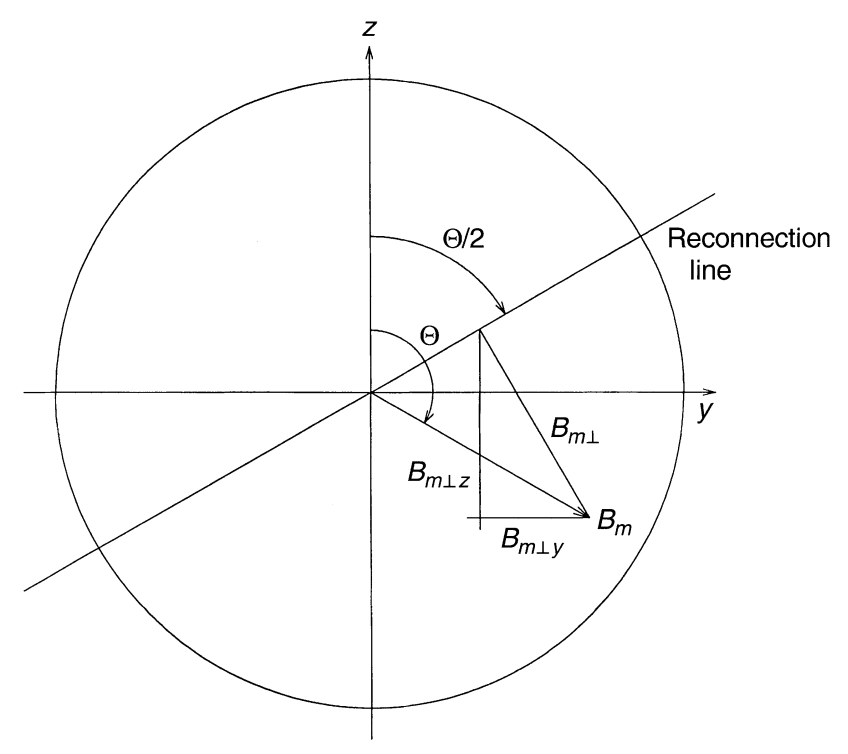

Fig. 1. Sketch for the calculation of the penetrating magnetic field components

$$
\begin{aligned}
B_{m \perp y} & =\alpha B_{m} \cdot \sin \frac{\Theta}{2} \cos \frac{\Theta}{2}=\frac{\alpha}{2} B_{m} \sin \Theta, \\
B_{m \perp z} & =-\alpha B_{m} \cdot \sin \frac{\Theta}{2} \sin \frac{\Theta}{2},
\end{aligned}
$$

where $\alpha$ is the coefficient of penetration, which has to be determined later.

It is seen from Eq. (3) that the $Z$-component of the penetrating magnetic field is always negative, regardless of the sign of the $Z$-component of the magnetosheath magnetic field, and tends to zero as $\Theta \rightarrow 0$.

Having substituted Eq. (3) into Eq. (2), one obtains:

$r_{0}=\left\{\frac{2.44 \cdot M_{E}^{\prime}}{\left[\sqrt{8 \pi P_{d}-\frac{\alpha^{2}}{4} B_{m}^{2} \sin ^{2} \Theta}+\alpha B_{m} \cdot \sin ^{2} \frac{\Theta}{2}\right]}\right\}^{1 / 3}$.

The last expression shows that the stand-off distance has a maximum at $\Theta=0$ and decreases with increasing $\Theta$. The rate of $r_{0}$ decrease depends on the value of the penetration parameter $\alpha$, which allows one to obtain that value by comparing experimental and model data.

\section{Experimental data and analysis}

According to Aubry et al. (1970), the magnetopause may be moving Earthward $1-2 \mathrm{~h}$ after the IMF southward turning. Therefore, to study the relationship between the magnetopause location and solar wind parameters, only those magnetopause crossings may be used which take place during relatively stable solar wind conditions. In this connection, for the analysis we selected 33 such events when the difference of the hourly mean values of the $Y$ - and $Z$-components of the IMF at the time of the crossing and $1 \mathrm{~h}$ before the crossing did not exceed $4 \mathrm{nT}$. And to eliminate dependence on the location of spacecraft within the magnetosheath, we used data obtained immediately upstream of the magnetopause in the vicinity of the subsolar point $\left(\varphi \leq 30^{\circ}, 09 \leq M L T \leq 15 \mathrm{~h}\right)$.

Table 1 presents the date and universal time of the crossing; the magnetopause stand-off distance $r_{0 \exp }$ (in Earth radii), the solar wind dynamic pressure $P_{d}$ (in dyn $\mathrm{cm}^{-2}$ ), the angles $\Theta_{w}$ in the solar wind and $\Theta_{m}$ in the magnetosheath, the intensity of the magnetosheath magnetic field $B_{\operatorname{mexp}}$ (in nT), its $Z$-component $\left(B_{m z}\right)$, the $Z$-component of the outer magnetospheric magnetic field $\left(B_{i z}\right)$ and the magnetosheath magnetic field in the magnetopause vicinity $B_{m t h}$ calculated according to the Pudovkin et al. (1995) model, respectively.

Concerning the values of $\Theta$, we have to notice the following. Most of the crossings listed in the table are picked up from the literature [the corresponding references are given in papers by Pudovkin (1982) and Pudovkin et al. (1995)]. For some of the published crossings, not all the necessary data were given. In particular, in many cases information on the direction of the magnetosheath magnetic field was not available. 
Table 1. List of magnetopause crossings

\begin{tabular}{|c|c|c|c|c|c|c|c|c|c|c|}
\hline $\mathrm{N}$ & date & UT & $\begin{array}{l}r_{0 \exp } \\
R_{E}\end{array}$ & $\begin{array}{l}P_{d} * 10^{8} \\
\text { dyn } \mathrm{cm}^{-2}\end{array}$ & $\begin{array}{l}\Theta_{w} \\
\text { deg. }\end{array}$ & $\begin{array}{l}\Theta_{m} \\
\text { deg. }\end{array}$ & $\begin{array}{l}B_{\operatorname{mexp}} \\
\mathrm{nT}\end{array}$ & $\begin{array}{l}B_{m z} \\
\mathrm{nT}\end{array}$ & $\begin{array}{l}B_{z i} \\
\mathrm{nT}\end{array}$ & $\begin{array}{l}B_{m t h} \\
\mathrm{nT}\end{array}$ \\
\hline 1 & 14.01 .67 & $1: 13$ & 6.6 & 10.5 & -154 & & 111 & -111 & 171 & 110 \\
\hline 2 & 09.03 .74 & $2: 52$ & 9.6 & 3.5 & -149 & -190 & 55 & -54 & 65 & 40 \\
\hline 3 & 17.03 .74 & $11: 16$ & 11.2 & 2.3 & 31 & 30 & 60 & 52 & 60 & 46 \\
\hline 4 & 05.11 .77 & $17: 15$ & 11.7 & 1.6 & 68 & 47 & 34 & 23 & 46 & 30 \\
\hline 5 & 24.11 .77 & $19: 36$ & 12.2 & 1.1 & 28 & 3 & 16 & 16 & 40 & 15 \\
\hline 6 & 15.08 .78 & $07: 14$ & 13.7 & 1 & -72 & & 20 & & & 12 \\
\hline 7 & 22.08 .78 & 09:07 & 11.3 & 2.3 & -58 & & 29 & & & 31 \\
\hline 8 & 27.08 .78 & $02: 46$ & 9.8 & 2.3 & 92 & 78 & 27 & 5 & 44 & 27 \\
\hline 9 & 29.08 .78 & $02: 17$ & 10 & 2.8 & -128 & & 43 & & & 48 \\
\hline 10 & 03.09 .78 & $07: 21$ & 10.5 & 1.9 & 141 & & 28 & & & 32 \\
\hline 11 & 05.09 .78 & $16: 12$ & 10.1 & 1.8 & 136 & 119 & 29 & -13 & 33 & 42 \\
\hline 12 & 08.09 .78 & $00: 43$ & 8.7 & 2.3 & 149 & 134 & 54 & -35 & 54 & 79 \\
\hline 13 & 10.09 .78 & $10: 51$ & 10 & 2.6 & -74 & -43 & 53 & 21 & 50 & 47 \\
\hline 14 & 12.09 .78 & $19: 56$ & 9.6 & 2.7 & 124 & 117 & 37 & -16 & 30 & 53 \\
\hline 15 & 15.09 .78 & $06: 56$ & 11.5 & 1.5 & 79 & & 35 & & & 27 \\
\hline 16 & 08.10 .78 & $18: 28$ & 9.5 & 3.5 & 67 & & 19 & & & 15 \\
\hline 17 & 11.10 .78 & $02: 37$ & 11.1 & 1.6 & -60 & & 41 & & & 33 \\
\hline 18 & 15.10 .78 & $21: 49$ & 10.6 & 1.8 & 108 & & 27 & & & 16 \\
\hline 19 & 01.11 .78 & $15: 21$ & 10.9 & 2.4 & 28 & 66 & 20 & 8 & 75 & 26 \\
\hline 20 & 15.11 .78 & $21: 52$ & 11.4 & 2.2 & 97 & 68 & 22 & 6 & 49 & 29 \\
\hline 21 & 25.11 .78 & $14: 14$ & 6.9 & 6.9 & 175 & 174 & 98 & -94 & 83 & 77 \\
\hline 22 & 08.09 .79 & $18: 31$ & 10.2 & 1.9 & 109 & 110 & 32 & -10 & 37 & 33 \\
\hline 23 & 11.09 .79 & 04:11 & 10.6 & 2.2 & 123 & 132 & 46 & -31 & 52 & 52 \\
\hline 24 & 15.09 .79 & $22: 04$ & 9.4 & 4.1 & -67 & -113 & 26 & -9 & 50 & 29 \\
\hline 25 & 04.11 .79 & $16: 38$ & 11.2 & 1.8 & 150 & 158 & 30 & -27 & 52 & 36 \\
\hline 26 & 29.08 .80 & $22: 29$ & 10.7 & 2 & -110 & -142 & 44 & -33 & 50 & 44 \\
\hline 27 & 01.09 .80 & $07: 53$ & 10.7 & 2.7 & -40 & -8 & 15 & 14 & 66 & 16 \\
\hline 28 & 08.09 .80 & $11: 34$ & 10.2 & 2 & 141 & 153 & 27 & -23 & 58 & 32 \\
\hline 29 & 13.09 .80 & $06: 31$ & 10.4 & 1.6 & 169 & 174 & 53 & -50 & 44 & 61 \\
\hline 30 & 15.09 .80 & $16: 25$ & 11.1 & 1.3 & -107 & -148 & 29 & -24 & 36 & 28 \\
\hline 31 & 18.09 .80 & $02: 15$ & 11.6 & 1.8 & -41 & -71 & 30 & 10 & 54 & 20 \\
\hline 32 & 27.09 .80 & $14: 02$ & 9.4 & 3.7 & -164 & -126 & 15 & -8 & 60 & 15 \\
\hline 33 & 06.11 .80 & $20: 29$ & 9.8 & 2.6 & -79 & -162 & 38 & -32 & 52 & 37 \\
\hline
\end{tabular}

Because of that and to make the set of data on the $\Theta$ angle more homogeneous, we listed values of the $\Theta$ angle within the solar wind (after King 1977, $1979,1986)$ instead of the angle $\Theta$ in the magnetosheath in Table 1. The regression analysis of data presented shows that the values of $\Theta_{m}$ are related to the values of $\Theta_{w}$ by the equality: $\Theta_{m}=-8^{\circ}+1.05 \Theta_{w}$; this allows us to hope that the error associated with the substitution of $\Theta_{w}$ is not too large.

Besides, for the crossing on 14 January 1967, only data on the $Z$-component of the magnetosheath magnetic field are available. However, as the angle $\Theta$ in this case is relatively large $\left(\Theta=-154^{\circ}\right)$ we use this value also as the $B_{m}$.

As is seen from the table, the entire set of data includes crossings with $|\Theta|$ varying from $30^{\circ}$ to $170^{\circ}, P_{d}$ varying from $10^{-8}$ to $10^{-7} \mathrm{dyn}^{-2}$, and $r_{0}$ from 6.6 to $13.7 R_{E}$.

Of course, the amount of data used for the analysis is limited. Nevertheless, we shall try to show that the main regularities of the $r_{0}$ behavior in dependence on the pressure $P_{d}$ and the angle $\Theta$ agree with known ones, which allows us to consider the data set given in the table as sufficiently representative.

The scatter plot of $r_{0 \exp }$ versus $\left(P_{d}\right)^{1 / 6}$ is shown in Fig. 2; the crossings corresponding to northward IMF
$\left(|\Theta|<70^{\circ}\right)$ are marked by open circles; those with southward $\operatorname{IMF}\left(|\Theta| \geq 110^{\circ}\right)$ are marked by solid circles, and crossings with intermediate angles $\Theta$ are marked by crosses. The solid and dashed lines represent the linear regression lines for crossings with southward and northward IMF, respectively.

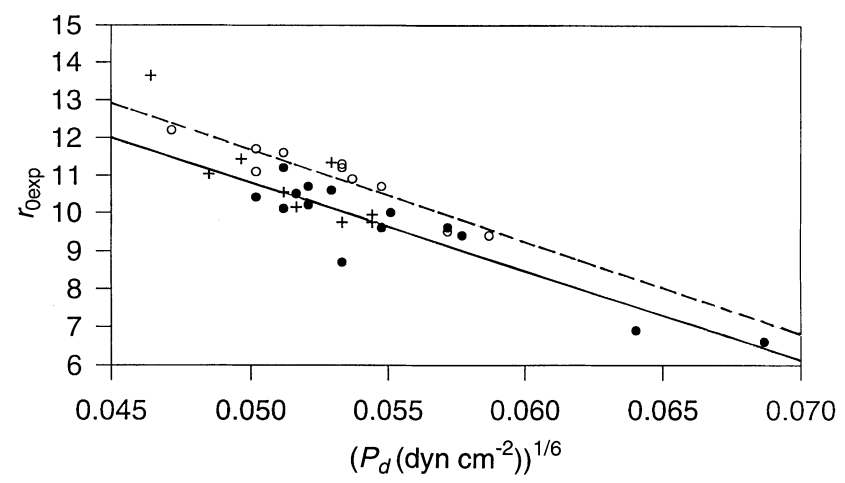

Fig. 2. Scatter plot of observed magnetopause stand-off distances versus $\left(P_{d}\right)^{1 / 6}$ for crossings with northward (open circles), intermediate (crosses), and southward (filled circles) IMF. Dashed and solid lines represent linear regression lines for crossings with northward and southward IMF orientation, respectively 
One can see in the figure a rather close relationship between $r_{0}$ and $\left(P_{d}\right)^{1 / 6}$ (coefficient of correlation equals 0.88 for the entire set of data), and the slope agrees with that obtained by Sibeck et al. (1991). Besides, it is seen that stand-off distances for the crossings with southward IMF are systematically less than those with northward IMF by approximately $1 R_{E}$, which also agrees with results by Fairfield (1971), Maezawa (1974), Sibeck et al. (1991), Petrinec et al. (1991), and Petrinec and Russell (1993). Thus, the set of data listed in the table is characterized by the same regularities as those obtained earlier in many studies, which permits us to consider it as sufficiently representative and to use it in the further analysis.

First of all we have to find the value of the Earth's effective magnetic momentum $M_{E}^{\prime}$. For this purpose, we selected crossings with $|\Theta| \leq 60^{\circ}$, and using Eq. (1) we obtained: $M_{E}^{\prime} / k=11.2 \cdot 10^{\overline{25}} \mathrm{G} \cdot \mathrm{cm}^{3}$, which results in $M_{E}^{\prime}=9.85 \cdot 10^{25} \mathrm{G} \cdot \mathrm{cm}^{3}=1.22 M_{E}$, with $k=0.88$ after Spreiter et al. (1968); the derived value is sufficiently close to that obtained by Schield (1969b): $M_{E}^{\prime}=1.25 M_{E}$.

According to Stern (1985), $M_{E}^{\prime}$ is independent of the solar wind pressure $P_{d}$; according to Petrinec and Russell (1993), $M_{E}^{\prime}$ does not depend on the DR-current intensity either; therefore, we shall suppose $M_{E}^{\prime}$ to be constant for all the crossings.

Having substituted the obtained value of $M_{E}^{\prime}$ into Eq. (1), one may calculate the values of the stand-off distance according to the Mead model $\left(r_{0 M}\right)$ for all the crossings under consideration. The results of this calculation are presented in Fig. 3, in which $r_{0 M}$ values are given versus experimental values $r_{0 \text { exp }}$ by open circles. As is seen in the figure, in spite of a close correlation between the parameters, the $r_{0 \exp }$ values prove to be less than the $r_{0 M}$ values, especially in the case of small $r_{0 \exp }$. We shall try to explain this disagreement later on by the

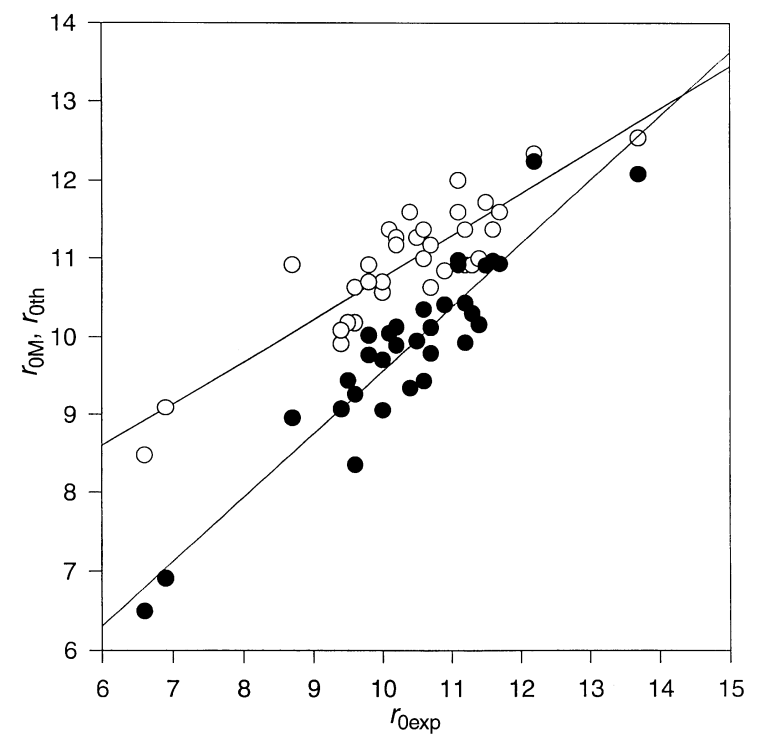

Fig. 3. Scatter plot of magnetopause stand-off distances calculated according to the Mead model (open circles) and the proposed model in this paper (filled circles). Thin lines represent linear regression lines influence of the magnetosheath magnetic field penetration into the magnetosphere.

In order to study the influence of the IMF on the magnetopause location, one has to eliminate the wellknown dependence of $r_{0}$ on the solar wind pressure. For this purpose we shall later on normalize all the measured values of $r_{0}$ by the values of $r_{0 M}$ calculated according to the Mead model, as in Pudovkin (1982). For the crossings under consideration, the ratio of $R_{0 \exp }=r_{\exp } / r_{0 M}$ varies in a rather wide range from 0.76 to 1.09 , and as the influence of the solar wind dynamic pressure is eliminated in the values of $R_{0 \text { exp }}$, this scatter of observed stand-off distances seems to be caused by the magnetosheath magnetic field penetrating into the magnetosphere.

To confirm this supposition, we calculated according to Eqs. (4) and (1) the normalized values of the model stand-off distances $R_{0 t h}=r_{0 t h} / r_{0 M}$ for various values of the parameter $\alpha$, and then studied the correlation between the $R_{0 \exp }$ and $R_{0 t h}$.

As a linear regression analysis shows, the coefficient of correlation between the model and experimental values of $R_{0}=r / r_{0 M}$ does not practically depend on the value of $\alpha$. At the same time, the regression coefficients $A$ and $C\left(R_{0 \exp }=A+C \cdot R_{0 t h}\right)$ are very sensitive to the value of $\alpha$ (Fig. 4).

One can deduce from Fig. 4 that the regression coefficients $C \approx 1$ and $A=0$ for $\alpha=1$; this means that the experimental and model values of $R_{0}$ coincide in the case when the magnetosheath magnetic field component perpendicular to the reconnection line totally penetrates into the dayside magnetosphere (or, in other words, the intensity of the magnetic field produced in the vicinity of the subsolar point by the three-dimensional current system existing in the cusp region equals the value of $\left.\boldsymbol{B}_{m \perp z L F}\right)$.

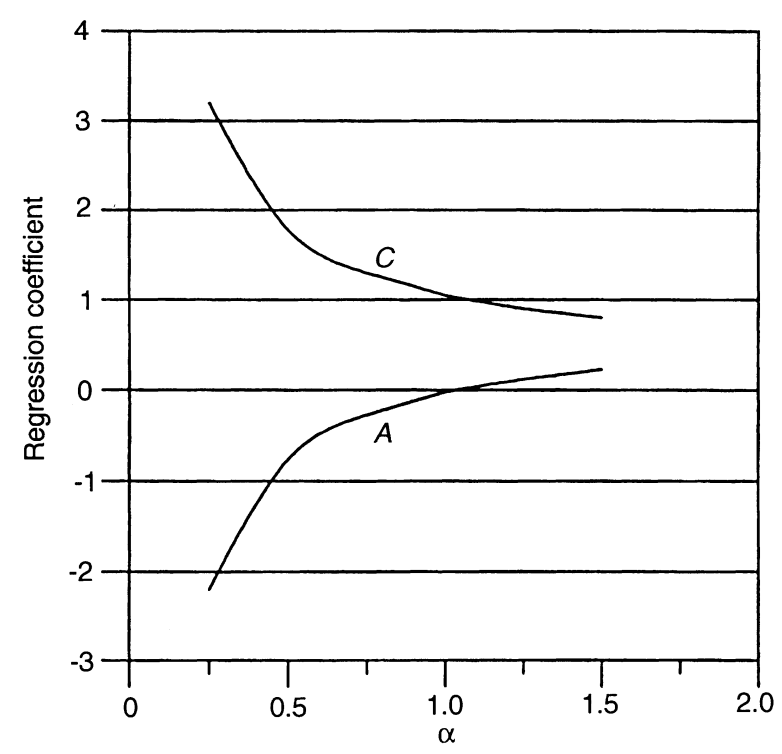

Fig. 4. Linear regression coefficients describing the relationship between $R_{0 \exp }$ and $R_{0 t h}$ values for different $\alpha$ 
And indeed, the scatter plot of $R_{0 \exp }$ versus $R_{0 t h}(\alpha=1)$ given in Fig. 5, illustrates a sufficiently close correlation between them $(r=0.73)$.

If the model is correct, and the magnetosheath magnetic field really penetrates into the dayside magnetosphere, the magnetospheric magnetic field measured in the vicinity of the subsolar point has to differ from that calculated in frame of the Mead model by the value of $\mathbf{B}_{m \perp}$ (Kovner and Feldstein, 1973; Pudovkin, 1982).

To check this supposition, we calculated the values of the $Z$-component of the magnetosheath magnetic field perpendicular to the reconnection line: $B_{m z} \approx-B_{m} \sin ^{2} \Theta / 2$, as well as the differences between the observed values of the magnetospheric magnetic field and those calculated according to the Mead model: $\delta B_{z}=B_{i z}-B_{z M}$. The scatter plot of $\delta B_{z}$ values versus $B_{m \perp z}$ values is given in Fig. 6a.

One can see in the figure that the values of $\delta B_{z}$ closely correlate with the values of the penetrating magneto-

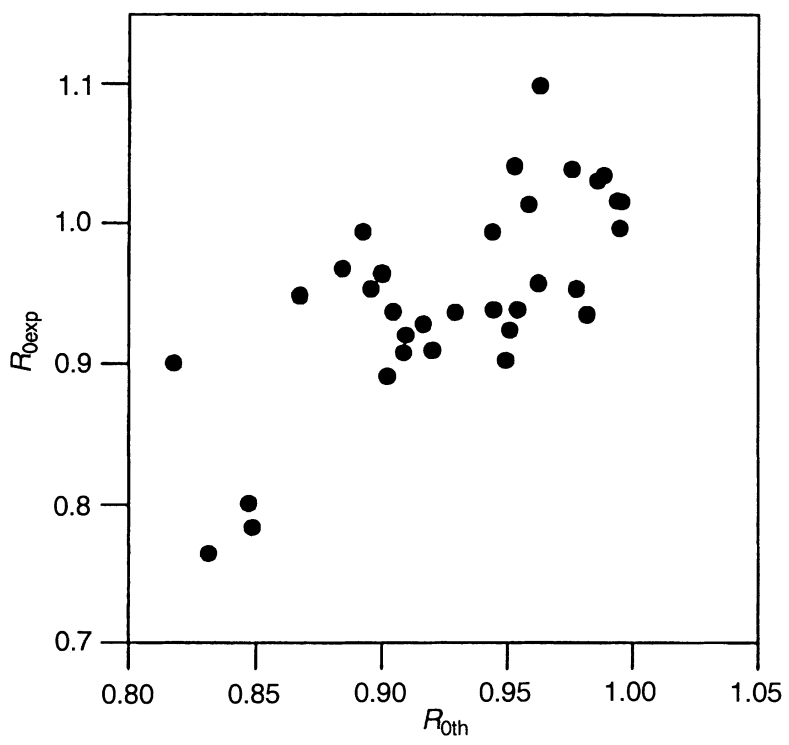

Fig. 5. Scatter plot of $R_{0 \exp }$ versus $R_{0 t h}$ values for $\alpha=1$ sheath magnetic field $(r=0.85)$. At the same time, the figure shows that the absolute values of the "observed" $\delta B_{z}$ are approximately twice as large as the magnetosheath magnetic field. This means that either the intensity of the penetrating magnetic field is really larger than the intensity of the magnetosheath magnetic field (e.g., $\alpha=1.5$ ), or the values of the Mead magnetic field are overstated, especially in cases of small $r_{0}$.

The first supposition seems to contradict the data presented in Fig. 4. Thus, let us consider the second possibility. As already mentioned, Petrinec and Russell (1993) have shown that for an average value of the solar wind pressure, the magnetopause stand-off distance does not depend on the Dst-field intensity, which supposes the magnetic momentum of the DR-currents to be independent of their intensity. This may be explained by a decrease in the mean radius of DRcurrents associated with the increase in their intensity, so that their momentum $M_{D R} \sim I_{D R} \cdot r_{D R}^{2}$ remains constant. However, this situation takes place only under mean conditions; when the solar wind pressure increases to such values that the magnetopause stand-off distance decreases to $6-7 R_{E}$, the radius of the DR-currents has also to decrease, which would result in decrease in their magnetic momentum.

In this connection we shall now suppose, in contrast to our previous consideration, that $M_{E} / k$ in Eq. (4) equals $11.2 \cdot 10^{25} \mathrm{G} \cdot \mathrm{cm}^{3}$ only when the solar wind pressure varies around its mean value of $2 \cdot 10^{-8} \mathrm{dyn} \mathrm{cm}^{-2}$, and essentially decreases when $P_{d}$ rises to approximately $10^{-7} \mathrm{dyn} \mathrm{cm}^{-2}$. The functional form of that dependence is not known, and we shall suppose, quite arbitrarily, only to show the general trend of $M_{E}^{\prime}$ decrease with $P_{d}$ increase, that it takes a form of

$$
\frac{M_{E}^{\prime}\left(P_{d}\right)}{k}=\left[8.1+3.1 \cdot \exp \left(\frac{10^{-8}-P_{d}}{3 \cdot 10^{-8}}\right)\right] \cdot 10^{25} \mathrm{G} \cdot \mathrm{cm}^{3} \text {. }
$$

By using the $M_{E}^{\prime}\left(P_{d}\right)$ values given by Eq. (5), one can recalculate the values of $r_{0 M}$ and afterwards the values of $B_{z M}\left(P_{d}\right)$ and $\delta B_{z}\left(P_{d}\right)$. The result of the calculations is
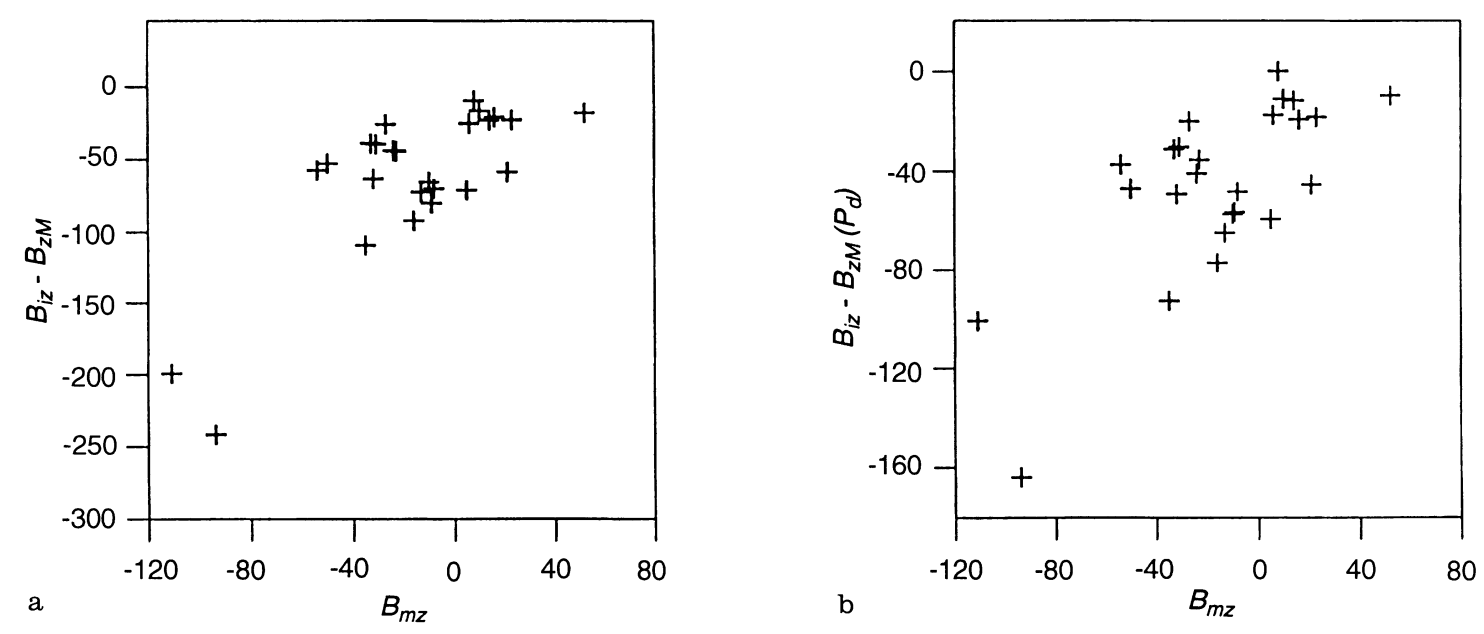

Fig. 6a, b. Scatter plots of $\delta B_{z}$ versus $B_{m z}$ values. a $\delta B_{z}=B_{i z}-B_{z M}$. b $\delta B_{z}=B_{i z}-B_{z M}\left(P_{d}\right)$ 
presented in Fig. 6b. As is seen in this figure, experimental and model values of $\delta B_{z}$ coincide much closer than in Fig. 6a, which seems to confirm the supposition regarding the dependence of the DR-current magnetic momentum on the value of the solar wind dynamic pressure.

In the preceding consideration we supposed, after Pudovkin et al. (1984), that it is the magnetosheath magnetic field component perpendicular to the merging line that penetrates into the dayside magnetosphere and causes the erosion of the magnetopause. In contrast to this, Sibeck et al. (1991) and Petrinec and Russell (1993) suggest that the intensity of the cusp-region Birkeland currents and the magnetopause erosion are determined by the $Z$-component of the solar wind (and hence of the magnetosheath) magnetic field.

In this connection, there are shown in Fig. 7a values of the magnetospheric magnetic field depression $\delta B_{z}$ versus the $Z$-component of the magnetosheath magnetic field. The correlation between the compared values is seen to decrease in this case: the coefficient of correlation equals $r=0.73$ compared to $r=0.85$ in Fig. 6a. Especially obvious disagreement between the experimental and model data is observed for northward IMF when $B_{m z}$ is positive and $\delta B_{z}$ negative. This disagreement vanishes when the model values of the penetrating magnetic field are calculated according to Eq. (3).

In Fig. $7 \mathrm{~b}$ the values of $\delta B_{z}$ versus $B_{m z}$ are presented for the case when the magnetospheric field intensity is calculated according to the Mead formula with $M_{E}^{\prime}$ depending on the solar wind dynamic pressure [Eq. (5)]. As is seen from the figure, the correlation between $\delta B_{z}$ and $B_{m z}$ in this case is better than in Fig. 7a. However, the disagreement between the observed and model values of the magnetospheric magnetic field for $B_{m z}>0$ still exists. This leads us to believe that it is the magnetosheath magnetic field component perpendicular to the reconnection line rather than its $Z$-component that influences the intensity of the Birkeland current loop in the dayside cusp region.

To obtain agreement between the model predictions and the experimental data, we had to suppose the
Earth's effective momentum $M_{E}^{\prime}$ to decrease with the increase of the solar wind dynamic pressure. However, if this supposition is correct, the normalizing factor $r_{0 M}$ in all the foregoing calculations is changing, and our conclusion on the value of the coefficient of penetration $\alpha$ may now be incorrect.

In this connection, we have repeated the regression analysis with new values of $r_{0 M}$ for the same values of $\alpha$ as in Fig. 4, and found that the coefficient $C$ is close to 1 and $A$ is close to zero in the case when $\alpha \approx 1$, as in our earlier calculations.

The scatter plot of experimental values of the magnetopause stand-off distance versus the model ones $\left(r_{0 t h}\right)$ with $M_{E}^{\prime}$ given by Eq. (5) and $\alpha=1$ is presented in Fig. 3 by solid circles. As is seen in the figure, the difference between the measured and model values of the stand-off distances at small $r_{0}$ has now disappeared, which confirms the model under consideration.

The data presented permit us to consider the model of the magnetopause erosion formulated in terms of the magnetosheath magnetic field penetration into the magnetosphere as quite adequate, and to accept Eq. (4) to predict the magnetopause stand-off distance.

However, the use of the magnetosheath magnetic field for the calculation of the value of $r_{0}$ is useless from the practical point of view, because when $B_{m}$ is known, $r_{0}$ is known too. In this connection, we will use now the results of the model by Pudovkin et al. (1995), which allows us to obtain values of $B_{m}$ from solar wind data: $v_{w}, n_{w}, B_{T}$, and $\Theta_{w}$.

The values of $B_{m}$ calculated according to that model $\left(B_{m t h}\right)$ are given in the last column of Table 1. Having substituted the $B_{m}$ values in Eq. (4) by $B_{m t h}$, one may calculate the magnetopause stand-off distance directly from the solar wind parameters $\left(r_{0 s w}\right)$. As in the preceding analysis, all the values were normalized by the values of $r_{0}$ calculated according to the Mead model with $M_{E}^{\prime}$ given by Eq. (5), and the results are presented in Fig. 8, where the values of $R_{0 \exp }$ are given versus the values of $R_{0 s w}=r_{0 s w} / r_{0 M}$. It is seen from the figure that the correlation between the experimental and model data is approximately the same as in Fig. $5(r=0.73)$.
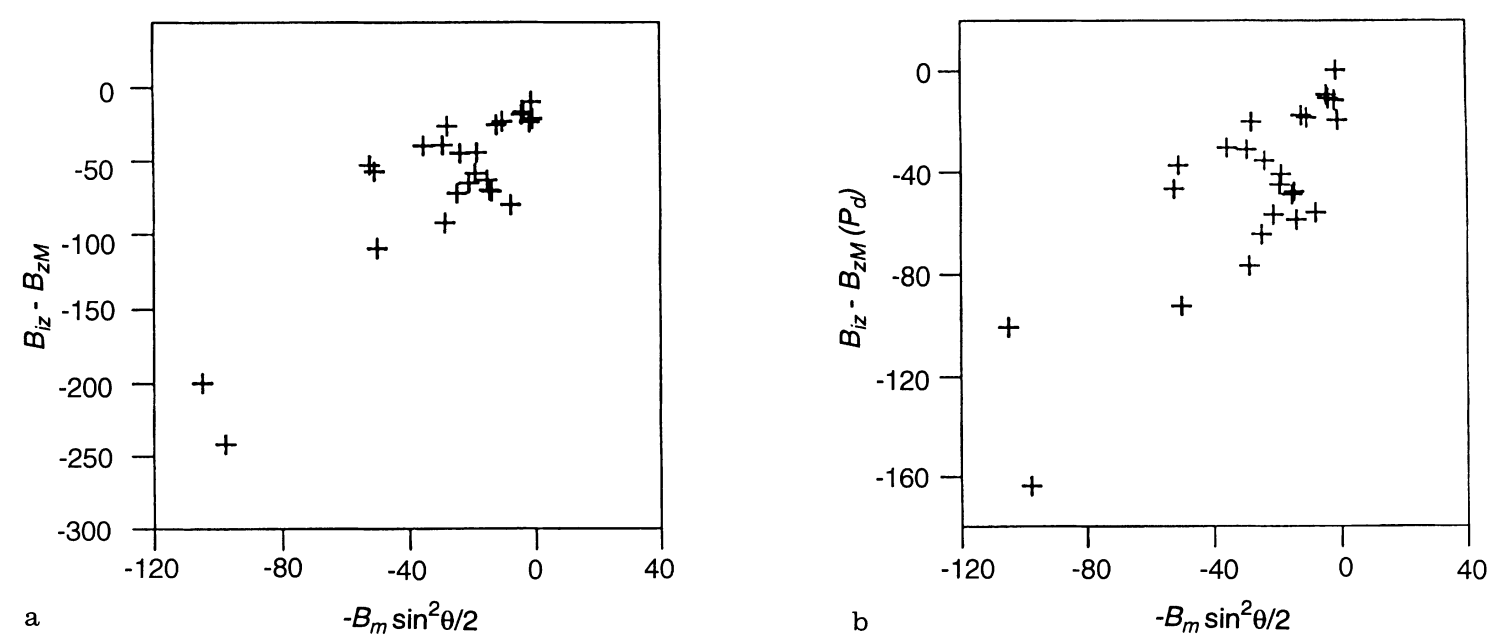

Fig. 7a,b. Scatter plots of $\delta B_{z}$ versus $B_{m \perp z}$ values. a $\delta B_{z}=B_{i z}-B_{z m}$. b $\delta B_{z}=B_{i z}-B_{z m}\left(P_{d}\right)$ 


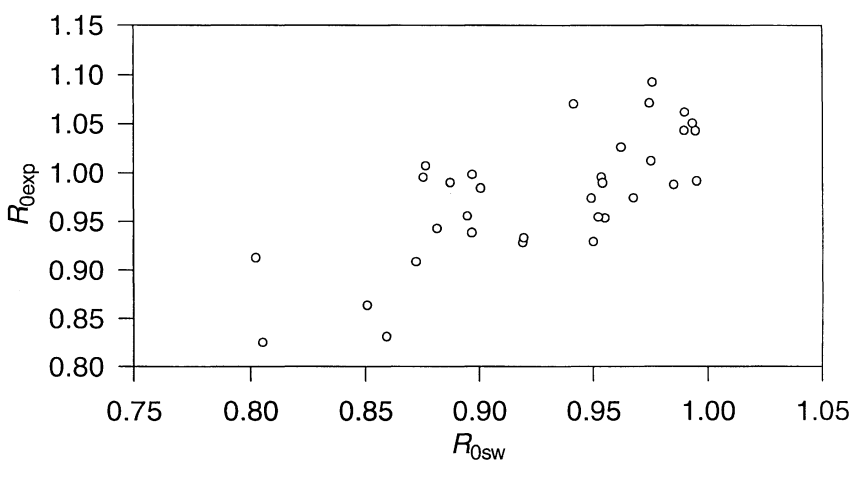

Fig. 8. Scatter plot of normalized values of the experimental magnetopause stand-off distances versus theoretical ones with $B_{m t h}$ calculated from solar wind parameters

So, we may hope that the stand-off distance values predicted from the solar wind data $\left(r_{0 s w}\right)$ in accordance with our model will also correlate with experimental ones sufficiently well.

And indeed, one can see in Fig. 9, where values of $r_{0 \exp }$ are given versus values of $r_{0 s w}$ a sufficiently close correlation between experimental and theoretical data $(r=0.93)$ with the slope coefficient being close to 1 .

\section{Conclusions}

Correlation studies of the type presented were carried out earlier by Holzer and Slavin (1978, 1979), Pudovkin (1982), Pudovkin et al. (1984), Sibeck et al. (1991), and Petrinec and Russell (1993). They showed that the magnetopause location is determined by the intensity of the southern component of the IMF, and that the magnetopause erosion is caused by the transfer of the magnetic flux from the dayside magnetosphere into the magnetotail. In this paper, we have tried to follow the entire chain of processes in the magnetosheath and in

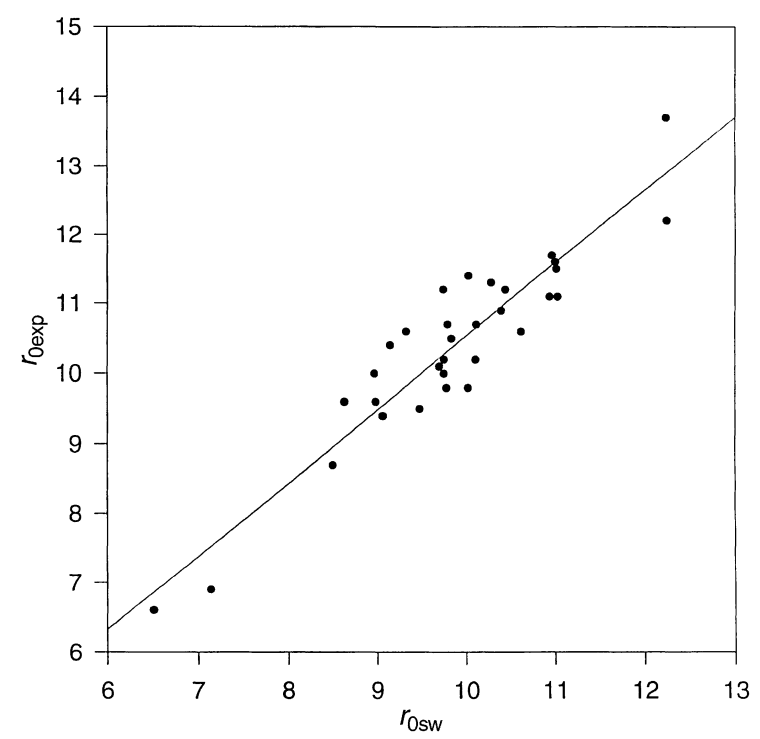

Fig. 9. Scatter plot of experimental magnetopause values of $r_{0}$ versus theoretical ones calculated from solar wind parameters the dayside magnetosphere which result in the Earthward displacement of the magnetopause during the periods of a southward IMF. So, first of all we have considered the magnetopause location and the intensity of the magnetospheric magnetic field depression in dependence on the magnetosheath magnetic field. This allowed us to estimate the intensity of the magnetic field produced by the Birkeland current loop in the cusp region in the magnetosphere.

The intensity and orientation of the magnetosheath magnetic field are determined by the parameters of the solar wind in front of the bow shock. A model by Pudovkin et al. $(1982,1995)$ allows one to calculate the magnetosheath magnetic field from the solar wind parameters. It has to be noted that according to the model, the value of $B_{m}$ depends not only on the IMF intensity and orientation, but also on the solar wind plasma density and velocity, which has not been taken into account by usual correlation analysis. Having revealed the relationship between the magnetopause location and the magnetosheath parameters, we have used the magnetosheath model proposed by Pudovkin et al. (1995) to predict the magnetopause stand-off distance directly from the solar wind parameters.

As a result, it was shown that:

1. In accordance with earlier results by Kovner and Feldstein (1973) and Pudovkin (1982), the Earthward displacement of the dayside magnetopause is associated with the depression of the outer magnetospheric magnetic field with respect to that calculated in the frame of the Mead (1964) model.

2. The difference between the observed values of the magnetospheric magnetic field and the Mead magnetospheric field is determined by the magnetosheath magnetic field intensity and direction. This allows one to explain the observed depression of the magnetospheric magnetic field after Kovner and Feldstein (1973) in terms of magnetosheath magnetic field penetration into the magnetosphere. The coefficient of field penetration $\alpha$ equals approximately 1 .

3. The effective magnetic momentum of the Earth, $M_{E}^{\prime} / k$, seems to depend on the dynamic pressure of the solar wind $P_{d}$, decreasing from $11.2 \cdot 10^{25} \mathrm{G} \cdot \mathrm{cm}^{3}$ at $P_{d} \approx 10^{-8}$ dyn $\mathrm{cm}^{-2}$ to $8-9 \cdot 10^{25} \mathrm{G} \cdot \mathrm{cm}^{3}$ with the increase in $P_{d}$ to approximately $10^{-7} \mathrm{dyn} \mathrm{cm}^{-2}$.

At this point we have to note that this result is obtained under the supposition of a constant penetration parameter $\alpha$ for all the crossings under consideration. In reality, this supposition may be incorrect, and the difference between the observed and model intensity of the magnetospheric magnetic field depression (see Figs. 6 and 7) may be explained by the increase in the parameter $\alpha$ with the increase in the solar wind pressure as well.

4. Of the two components of the magnetosheath magnetic field, $B_{m z}$ and $B_{m \perp z}$, the latter seems to be more closely correlated with the intensity of the outer magnetosphere magnetic field depression.

5. Combining the magnetosheath model by Pudovkin et al. and the magnetosheath penetration model 
presented here, one can predict the magnetopause stand-off distance from solar wind data.

A close correlation between the predicted and experimental data allows us to consider Eq. (4) with $B_{m}$ calculated from the solar wind parameters as quite acceptable, and thereby confirms on the whole the combined model discussed.

It has to be remembered here that the model presented is based on the supposition on the subsolar point reconnection, and as this takes place most probably for $|\Theta|>50^{\circ}$ (Pudovkin and Semenov, 1989), the model may be inadequate for cases with extremely northward IMF.

Acknowledgements. This work was supported by grants of the Soros Foundation, No. 837 P, Russian Fundamental Science Foundation N 97-05-64458, and the "Jubiläumsfonds der Österreichischen Nationalbank", No. 5140. MIP and SAZ thank the "Österreichische Akademie der Wissenschaften" in Vienna and the "Institut für Weltraumforschung" at Graz for the financial support during their visits to Graz.

Topical Editor K.-H. Glaßmeier thanks two referees for their help in evaluating this paper.

\section{References}

Aubry, M. P., C. T. Russell, and M. G. Kivelson, Inward motion of the magnetopause before a substorm, J. Geophys. Res., 75, 7018-7031, 1970

Chapman, S., and V. C. A. Ferraro, A new theory of magnetic storms, Terr. Magn. Atmos. Electr., 36, 77-97, 171-186, 1931.

Fairfield, D. H., Average and unusual locations of the Earth's magnetopause and bow shock, J. Geophys. Res., 76, 6700-6716, 1971.

Holzer, R. E., and G. C. Reid, The response of the day-side magnetosphere-ionosphere system to time-varying field line reconnection at the magnetopause: 1 . theoretical model, $J$. Geophys. Res., 80, 2041-2049, 1975.

Holzer, R. E., and J. A. Slavin, Magnetic flux transfer associated with expansions and contractions of the dayside magnetosphere, J. Geophys. Res., 83, 3831-3839, 1978.

Holzer, R. E., and J. A. Slavin, A correlative study of magnetic flux transfer in the magnetosphere, J. Geophys. Res., 84, 2573-2578, 1979.

King, J. H., Interplanetary medium data book, NSSDC, Greenbelt, 1977, 1979, 1986

Kovner, M. S., and Ya. I. Feldstein, On solar wind interaction with the Earth's magnetosphere, Planet. Space Sci., 21, 1191-1211, 1973.

Maezawa, K., Dependence of the magnetopause position on the southward interplanetary magnetic field, Planet. Space Sci., 22, 1443-1453, 1974

McPherron, R. L., C. T. Russell, and M. P. Aubry, Satellite studies of magnetospheric substorms on August 15, 1968: I. phenomenological model for substorms, J. Geophys. Res., 78, 31313149,1973
Mead, C. D., Deformation of the geomagnetic field by the solar wind, J. Geophys. Res., 69, 1181-1195, 1964.

Petrinec, S. P., and C. T. Russell, External and internal influences on the size of the dayside terrestrial magnetosphere, Geophys. Res. Lett., 20, 339-342, 1993.

Petrinec, S. P., P. Song, and C. T. Russell, Solar cycle variations in the size and shape of the magnetopause, J. Geophys. Res., 96, 7893-7896, 1991.

Pudovkin, M. I., Parameters of the day side magnetopause and generation of the electric field in the magnetosphere, Ann. Geophys., 38, 745-753, 1982.

Pudovkin, M. I., and V. S. Semenov, The role of the magnetic field reconnection in the solar wind - magnetosphere interaction, in Proc. Int. Worksh. Reconnection in space plasma, ESA-SP 285, Vol. II, pp. 281-286, 1989.

Pudovkin, M. I., M. F. Heyn, and V. V. Lebedeva, Magnetosheath's parameters and their dependence on intensity and direction of the solar wind magnetic field, J. Geophys. Res., 87, 8131-8138, 1982.

Pudovkin, M. I., T.V. Kuznetsova, and S. A. Zaitseva, Location of the dayside magnetopause in dependence on the IMF direction (in Russian), Magnetosph. Res., N3, "Radio i Svyaz", 14-24, 1984.

Pudovkin, M. I., N. A. Tsyganenko, and A. V. Usmanov, Influence of field-aligned currents on the structure and location of polar cusps, Geomagn. Aeron., 26, 801-804, 1986a.

Pudovkin, M. I., S. A. Zaitseva, and A. V. Usmanov, Field-aligned current distribution in the polar cusp region in dependence on the IMF orientation, Geomagn. Aeron., 26, 650-654, 1986b.

Pudovkin, M. I., S. A. Zaitseva, and B. P. Besser, Magnetopause magnetic barrier parameters in dependence on the solar wind magnetic field orientation, Ann. Geophysicae, 13, 828-835, 1995.

Reid, G. C., and R. E. Holzer, The response of the day-side magnetosphere-ionosphere system to time-varying field line reconnection at the magnetopause: 1. erosion event of March 27, 1968, J. Geophys. Res., 80, 2050-2056, 1975.

Schield, M. A., Pressure balance between solar wind and the magnetosphere, J. Geophys. Res., 74, 1275-1286, 1969a.

Schield, M. A., Correction to paper by M.A. Schield "Pressure balance between solar wind and the magnetosphere", $J$. Geophys. Res., 74, 5189-5190, 1969b.

Sibeck, D. G., R. E. Lopez, and E. C. Roelof, Solar wind control of the magnetopause shape, location, and motion, J. Geophys. Res., 96, 5489-5495, 1991.

Spreiter, J. R., and B. R. Briggs, Theoretical determination of the form of the boundary of the solar corpuscular stream produced by interaction with the magnetic dipole field of the Earth, $J$. Geophys. Res., 67, 37-51, 1962.

Spreiter, J. R., A. Y. Alksne, and A. L. Summers, External aerodynamics of the magnetosphere, in Physics of the magnetosphere, Eds. R.L. Corovillano, J.F. McClay, and H.R. Radoski, D. Reidel, Dordrecht, pp. 301-364, 1968.

Stern, D. P., Parabolic harmonics in magnetospheric modeling: the main dipole and ring current, J. Geophys. Res., 90, 1085110863, 1985.

Tsyganenko, N. A., and D. G. Sibeck, Concerning flux erosion from the dayside magnetosphere, J. Geophys. Res., 99, 13425-13436, 1994.

Yeh, T., Day-side reconnection between a dipolar geomagnetic field and a uniform interplanetary field, J. Geophys. Res., 81, 2140, 1976. 